WI - Editorial

\title{
Reformen muss man einleiten, wenn es wirtschaftlich gut geht - also spätestens jetzt!
}

Die Autoren
Hans Ulrich Buhl
Wolfgang König
Prof. Dr. Hans Ulrich Buhl
Universität Augsburg
Lehrstuhl für Betriebswirtschaftslehre,
Wirtschaftsinformatik \&
Financial Engineering
Universitätsstraße 16
86135 Augsburg
hans-ulrich.buhl@wiwi.uni-augsburg.de
Prof. Dr. Wolfgang König
Universität Frankfurt
Institut für Wirtschaftsinformatik
Mertonstraße 17
60054 Frankfurt am Main
koenig@wiwi.uni-frankfurt.de

Die Arbeitsagentur meldet Ende September die niedrigste Arbeitslosigkeit seit 12 Jahren und die höchste Beschäftigung seit der Wiedervereinigung, in prosperierenden Regionen steigen die Mieten und finden viele Arbeitgeber auf ihre offenen Stellen trotz auch real wieder steigender Löhne und Gehälter keine Arbeitssuchenden mehr. Die Finanzbehörden melden Rekordergebnisse bei den Steuereinnahmen, der vierfache EU-Stabilitätspaktsünder Deutschland kann aufgrund der Überschüsse in den Kommunal- und Länderhaushalten sowie der Sozialversicherung zum ersten Mal seit 40 Jahren 2007 wieder einen ausgeglichenen Haushalt nach Brüs- sel melden. Die weltweite wirtschaftliche Dynamik sorgt beim Warenexportweltmeister Deutschland einmal mehr für eine gute Konjunktur, die Politiker sprechen davon, dass der Aufschwung bei den Menschen ankommt. Alles bestens, die Reformen greifen, frohe Weihnachten, weiter so?

Schauen wir etwas genauer hin und gießen etwas Wasser in den Wein: Betrachtet man die Wachstumsraten in den letzten Jahren und extrapoliert diese in die $\mathrm{Zu}$ kunft, ergibt sich folgendes Bild: Die wirtschaftliche Dynamik ist bei Dienstleistungen sehr viel höher als bei Warenexporten, daher wachsen andere Länder mit stärkerem Dienstleistungsexport schneller und stabiler als der Dienstleistungsnettoimporteur Deutschland. Den Titel des Warenexportweltmeisters verliert Deutschland in wenigen Jahren an das sehr viel schneller wachsende China. Die deutsche Volkswirtschaft verliert also trotz der zur Zeit guten Zahlen weiterhin Marktanteile an schneller wachsende Länder wie China und Indien, aber auch an dienstleistungsintensivere Industrienationen mit ihren stark wachsenden Dienstleistungssektoren wie USA und UK. In den OECD-Ländern sind mittlerweile über $70 \%$ der Arbeitnehmer im Dienstleistungssektor beschäftigt - mit weiter steigender Tendenz. Die EZB meldete 2007, dass im Euro-Währungsraum seit 1999 12,9 Mio. neue Stellen entstanden sind, davon fast alle im Dienstleistungssektor. Deutschland liegt aber im Bereich der Dienstleistungen sowohl beim Produktivitätswachstum als auch bei der Beschäftigungsentwicklung nicht an der Spitze, sondern nur im Mittelfeld. Und das, obwohl uns unsere Statistiken sagen, dass über die letzten 10 Jahre gerade die wissensintensiven Dienstleistungen und speziell ITDienstleistungen - der new economy und ihrer Krise zum Trotz - unsere stärksten Wachstumsmotoren waren und sind.
Wenn wir als Volkswirtschaft unsere Marktanteile verteidigen oder gar steigern wollen, ist daher klar, was zu tun ist: Wir dürfen auf betriebs- und volkswirtschaftlicher Ebene den Wandel zur Dienstleistungs- und Wissensgesellschaft nicht verzögern, sondern müssen ihn in einer konzertierten Aktion von Politik, Wirtschaft und Wissenschaft im Gegenteil beschleunigen. Dabei müssen wir uns auf die wachstumsstarken Bereiche konzentrieren, in denen wir trotz unseres Lohnniveaus komparative Vorteile aufweisen. Dies gilt einerseits für die produktnahen Dienstleistungen, in denen wir auf unsere traditionellen Produktstärken bspw. im Maschinen- und Automobilbau aufsetzen können. Während dort früher der Produktverkauf im Vordergrund stand und die Dienstleistung begleitenden Charakter hatte, sehen wir vielfach eine klare Bedeutungsumkehr: Die Differenzierung in der Dienstleistungsinnovation ist erfolgsentscheidend, das Produkt bekommt commodity-Charakter, wird austauschbar und vom Wertschöpfungsanteil her immer geringfügiger. Komparative Vorteile haben wir aber andererseits in der Gestaltung und im Management komplexer Systeme. Erfolgsstorys wie die von SAP zeigen das Potenzial, welches dadurch erschließbar ist. Bei komplexen Systemen von Kernkraftwerken bis hin zur Magnetschwebebahn haben wir in der Vergangenheit durch jahrzehntelange fruchtlose Diskussionen viele wirtschaftliche Chancen nicht genutzt und Wachstum an Wettbewerber verloren. Bei Projekten wie Galileo und den darauf aufbauenden Dienstleistungsinnovationen droht uns ähnliches. Wenn wir im internationalen Wettbewerb bestehen und zukünftiges Wirtschaftswachstum ermöglichen wollen, darf uns dies im schnell wachsenden Bereich wissensintensiver Dienstleistungen nicht ein weiteres Mal passieren. Gerade IT-basierte Dienstleistungen und die Gestaltung und 
Beherrschung der damit entstehenden komplexen Wertschöpfungsnetze bietet der deutschen Volkswirtschaft beträchtliche Wachstumschancen. Der globale Wettbewerb verzeiht dort auch in Zukunft keine Fehler, wie wir in vielen IT-Bereichen in den letzten Jahrzehnten schmerzhaft erfahren mussten.

Was machen wir heute falsch? Statt der notwendigen Anreize zum Wirtschaftsumbau sehen wir von der Wirtschaftspolitik nach wie vor zu viele Erhaltungssubventionen, lassen die Gewerkschaften Lohnzugeständnisse nicht dort zu, wo diese Investitionscharakter zur Erschließung neuer Märkte haben, sondern nur dort, wo es Unternehmen schlecht geht. Oft ist die Ursache dafür, dass den Unternehmen die Transformation $\mathrm{zu}$ den Dienstleistungen nicht rechtzeitig gelungen ist. Auch dieses Verhalten der Tarifpartner bremst den notwendigen Wandel, statt ihn zu beschleunigen. Die meisten Wissenschaftsdisziplinen konzentrieren sich - wie der Name schon besagt - auf ihre disziplinären Wurzeln statt auf die interdisziplinären Chancen und $\mathrm{zu}$ einem viel $\mathrm{zu}$ großen Anteil weitgehend auf den industriellen Wirtschaftssektor. Auch hier ist ein mangelnder Wandel hin zur Dienstleistungsforschung zu konstatieren. Und die wenigen Aktivitäten finden zudem in unterschiedlichen Fachgebieten meist isoliert voneinander statt. Die Forschungsförderung sollte mit Blick auf die wirtschaftlichen Chancen hier klare Impulse geben. Doch was sehen wir? Forschungsgelder werden nach wie vor sehr viel stärker in Produkte und nur zum kleinen Teil in Dienstleistungen investiert. So stellt der Bund im Rahmen der HightechStrategie nur 50 Mio. $€$ für das Gebiet innovativer und interdisziplinärer Dienstleistungen, aber 3,65 Mrd. € für Raumfahrttechnologie zur Verfügung. $\mathrm{Zu}$ recht kritisiert daher die Opposition etwas pointiert: „Wer den größten Teil seiner Hochtechnologiemittel in die Raumfahrt investiert, hat die Notwendigkeiten auf Erden falsch eingeschätzt" (Bündnis 90/Grüne) bzw. „Wir investieren unsere Forschungsgelder zu stark in unsere wirtschaftliche Vergangenheit und $\mathrm{zu}$ wenig in unsere wirtschaftliche Zukunft" (FDP).

Was also müssen wir jetzt besser machen? Die Haushalte von Bund, Ländern und Kommunen lassen wieder Gestaltungsspielräume zu. Vielen Unternehmen, die in den letzten Jahren die Weichen richtig gestellt haben, geht es gut. Und auch die Hochschulen haben aufgrund der Studiengebühren in vielen Bundesländern und des Ausbaus der Studienplätze zur Bewäl- tigung der hohen Studentenzahlen des nächsten Jahrzehnts wieder mehr Stellen und Mittel zur Verfügung. Die Investition dieser Mittel wird - im Guten wie im Schlechten - für unsere wirtschaftliche $\mathrm{Zu}$ kunft entscheidend sein, eine zweite solche Chance werden wir bei suboptimaler Investition und demzufolge künftig fehlenden Mitteln aufgrund der demographischen Entwicklung bei danach rückläufigen Studentenzahlen nicht bekommen.

Ein integrierter Ansatz zur Zusammenarbeit von Politik, Wirtschaft und Wissenschaft zur interdisziplinären Gestaltung und wirtschaftlichen Nutzung von Dienstleistungsinnovationen muss bewirken, dass einerseits die oben bereits genannten Potenziale genutzt werden und diese andererseits einen Beitrag zu den nachfolgenden Punkten leisten:

(1) Umgestaltung bestehender Ausbildungssysteme vom Kindergarten bis zur Hochschule zur Vorbereitung auf unsere Innovationschancen in der Dienstleistungs- und Wissensgesellschaft und der Transformation unserer technologischen Basis. Für die Hochschulen bedeutet das, ihre Studiengänge auf den Prüfstand zu stellen und auf die zukünftigen Anforderungen neu auszurichten - dies gilt auch für die Ingenieurwissenschaften, die dadurch für Abiturienten attraktiver gemacht werden können. Konflikte mit vielen beamteten Besitzstandswahrern werden dabei unausweichlich sein.

(2) Neue Studienplätze dürfen nur dort entstehen, wo die Hausaufgaben zur Vorbereitung auf die wirtschaftlichen und gesellschaftlichen Herausforderungen von morgen zuvor erledigt worden sind. Es geht um weit mehr als darum, die Abiturienten ein paar Jahre weg von der Straße und hin zu den Hochschulen zu bringen.

(3) Studienplätze in „billigen“ Disziplinen ohne hohe wirtschaftliche Chancen zu schaffen wird langfristig volkswirtschaftlich extrem teuer. Stattdessen sollten diese gerade dort geschaffen werden, wo die Dienstleistungsherausforderungen angenommen werden und die bisherigen, gegenwärtigen und künftigen Berufschancen der Absolventen dies rechtfertigen - auch wenn diese kurzfristig teurer sind. Gerade interdisziplinäre Studiengänge wie Wirtschaftsinformatik und Wirtschaftsingenieurwesen haben dies bisher gezeigt und haben bei entsprechender Weiterentwicklung auch künftig beste Chancen, dies nachzuweisen.
(4) Die Wirtschaft darf sich nicht auf die Position zurückziehen, dass sie mit der Zahlung der Steuern ihre Pflicht erfüllt hat, sondern muss die Weiterentwicklung des Ausbildungssystems im Sinne dieser drei Punkte aus eigenem Interesse mitgestalten. Sonst wird morgen erneut der „war for talent" beklagt.

(5) Parallel zu diesen Ausbildungsanstrengungen muss - wie die o. g. Beispiele zeigen - auch die Forschungsförderung viel konsequenter auf die diskutierten wirtschaftlichen Herausforderungen mit klarem Dienstleistungsund Transformationsfokus ausgerichtet werden. Und nur so können die Hochschulen in Forschung und Lehre ihrer eigenen Transformationsaufgabe gerecht werden.

(6) Durch diese Maßnahmen wird auch die Zusammenarbeit der Wissenschaft mit den Unternehmen zur Bewältigung von deren Herausforderungen gefördert.

Was kann und muss die Zeitschrift WIRTSCHAFTSINFORMATIK hierzu beitragen? Wir setzen uns in zunehmendem Maße mit den Wirtschaftsinformatikaspekten der Dienstleistungstransformation auseinander. So widmen wir bspw. dem Thema "Serviceorientierte Architekturen und Webservices" das Schwerpunktheft 1/2008, dabei werden wir auch auf das Thema Service Science und die spezifischen Charakteristika von Dienstleistungen und ihre Bedeutung für die Wirtschaftsinformatik eingehen. Dem Thema „Hybride Wertschöpfung - Integration von Produkt und Dienstleistung" widmet sich aus den o. g. Gründen produktnaher Dienstleistungen das Schwerpunktheft 3/2008.

Mit dem Verlag diskutieren die Herausgeber intensiv, wie wir das Wissen aus unserem interdisziplinären Fachgebiet künftig noch besser Ihnen, unseren Lesern, die Sie auch zu einem immer größeren Anteil im Dienstleistungsbereich unterwegs sind, noch kundengerechter online und gedruckt zur Verfügung stellen können. Und natürlich rufen wir unsere Autoren aus Wissenschaft und Praxis gerne auf, uns künftig noch mehr Beiträge zuzusenden, die sich mit Wirtschaftsinformatik und Dienstleistungen beschäftigen. Dienstleistungsinnovationen sind und bleiben in unserem Fokus.

Prof. Dr. Hans Ulrich Buhl Prof. Dr. Wolfgang König 